\title{
Assistance in Creation of Project Solutions by Means of System-Object Determinant Analysis
}

\author{
V.V. Mikhelev ${ }^{1}$ and S.I. Matorin ${ }^{2}$ \\ ${ }^{1}$ Belgorod State National Research University, Belgorod, Russia, 308015 \\ ${ }^{2}$ Belgorod University of Cooperation, Economics and Law, Belgorod, Russia, 308023 \\ keeper121@ya.ru, matorinesoftconnect.ru
}

\begin{abstract}
This paper contains a description of the original system-object determinant analysis (SODA) which allows you to formalize the process of developing complex technical systems. In SODA the developing process is presented as a procedure for defining the required properties of the system in the form of a sequential classification of conceptual and material systems. The paper presents the stages of sequential construction of generic, genetic and partitive classifications of a given subject area. It is shown that these classifications make it possible to unambiguously determine the external determinant of the system (functional requirements of the supersystem) and its internal determinant (functional properties of the system that ensure the fulfillment of the requirements imposed by the supersystem).
\end{abstract}

Keywords: System-Object Determinant Analysis, Generic Classification, Genetic Classification, Partitive Classification, System-Object Approach "UnitFunction-Object", Descriptive Logic, System-Class, System-Phenomenon.

\section{Introduction}

Successes of analytical activity under the slogan of systems analysis have shown its usefulness and effectiveness for solving complex practical problems. However, at present «there is no unambiguity in the understanding of the system analysis itself» $[1, p$. 231]. There are several definitions of system analysis which are a set of different principles approaches and methods but in practice they do not include the actual system analytics [1].

In the specialized literature on systems theory it is noted that «there are no wellestablished systems analysis technologies in practice» [2]. The existing technologies cannot even be categorized unambiguously [3]. A sufficiently detailed review according to the authors of systems analysis technologies is presented, for example, in works $[4,5]$. At the same time, the authors believe that the existence of various and inconsistent technologies of system analysis is due to specific still unresolved methodological problems of the traditional systems approach and system analysis [6]. In addition, the procedures of all the methods and technologies of system analysis existing up to the present time do not have a formal algorithmic description. 
There are a lot of papers describing methods of system analysis (Quaid [7], Optner [8], Chernyak [9], Golubkov [10], Yang [11], Tarasenko [12], etc.). However, you can see that they are all different general guidelines the implementation of which is not specifically indicated. But most importantly, all these methods, in fact, do not take into account the concept of "system", do not take into account the systemic effect, do not use a principled approach and do not rely on general system laws.

To solve these problems, the article [13] proposes a new original system analysis tool based on the system-object approach - the conceptual apparatus of system-object determinant analysis (SODA). It provides a researcher an effective and versatile toolkit for the analysis and design of complex weakly formalized systems.

The method of system analysis proposed in [13] in contrast to those mentioned above is a formalized procedure that takes into account the systemic effect and some general systemic patterns.

SODA includes three stages. Firstly, the identification of the class to which the analyzed or projected system belongs which is carried out when constructing a taxonomic (generic) classification of the subject area. This allows the researcher to determine the external determinant of the system. Functional request of a higher order system (supersystem) for a system with a given function. Secondly, tracing the stages of formation or creation of a system which is carried out when constructing a genetic (stadial) classification of a selected class of systems. This allows, on the one hand to specify the requirements for the system and on the other hand to unambiguously determine the internal determinant of the system. Its actual functionality arising under the influence of an external determinant. Third, the decomposition of the requirements for the system as a phenomenon, which is carried out when constructing a partitive (whole-part) classification of the system or its meronomy. This gives an idea of the ways to ensure the correspondence of the subsystems of the analyzed or developed system to its internal determinant (and in the limit external), i.e. about the ways of functioning or construction of the system.

The system-object determinant analysis proposed in [13] which is a refinement and concretization of the ideas presented in [14] can be used as a working useful toolkit in the design of complex information and technical systems.

\section{Formalized Description of System-Object Determinant Analysis}

Descriptive logic (DL) is used in the form ALCOIQ(D) [15] for a formalized description of SODA procedures. The syntax of this DL is briefly represented as the following expression:

$$
\left\{\mathrm{T}, \perp, A, A \sqsubseteq C, \neg C, C \sqcap D, C \sqcup D, \exists R . C, \forall R . C, \geq \mathrm{n} R . C,\{a\}, \exists\left[u_{1}, \ldots, u_{n}\right] . P\right\}
$$

Here the symbols T and $\perp-$ concepts (true and false). A - atomic concept, C, D general concepts; $\mathrm{R}-$ atomic role. $\{\mathrm{a}\}-$ nominal; $\exists\left[u_{1}, \ldots, u_{n}\right] . P-$ the concept of a specific area; $P$ - predicate symbol; $u_{1}, \ldots, u_{n}$ - attributes. The DL also uses the following terms: TBox is a set of terminological axioms; RBox is a set of axioms for roles 
and their relationships; ABox is a set of axioms for individuals and their relationships. The sum of the above sets of axioms and defines the subject area $\mathrm{K}=$ TBox $\cup$ RBox $U$ ABox.

The system-object approach "Unit-Function-Object" [16] describes systems-classes (conceptual systems according to Ackoff or external systems according to Schrader) by means of DL in the following form:

$$
S_{i, j}{ }^{l}=S_{i-1, l^{p}} \sqcap \exists R S_{i, j}{ }^{l},
$$

$S_{i, j}{ }^{l}, S_{i-1, l^{j}}, R S_{i, j}{ }^{l}$ - abstract classes (concepts), where $i=0 \ldots N, i$ - hierarchy tier number; $j$ - node number within one tier of the hierarchy; $l, p$ - parent node numbers for the current node in the hierarchy level.

A system that is a concrete system-class (consisting of instances, not subclasses) in terms of DL is described as a set of three component elements "Unit-Function-Object" (UFO-elements) [16]:

$$
S=[L ? \sqcup L ! ; L ! \sqcap \exists R S . L ? ; O S ? \sqcup O S ! \sqcup O S f],
$$

where $L$ ? $\sqcup L$ ! - node that is the intersection of inputs $L$ ? and outputs $L ! ; L ! \sqcap \exists R . L$ ? function which converts inputs to outputs; $O s ? \sqcup O s ! \sqcup O s f$ - an object that implements a function and describes substantial characteristics.

Systems-phenomena (material systems according to Ackoff or internal systems according to Schraider), by means of DL (concepts of nominal value and specific areas) can be represented in the following form:

$$
s=\left[\{\mathrm{L} ?\} \sqcup\{\mathrm{L} !\} ;\{\mathrm{L} !\} \sqcap \text { GhasRelation. }\{\mathrm{L} ?\} ; \mathrm{OS} ?=\mathrm{n}_{\mathrm{n} 1} \sqcup \mathrm{OS} !=_{\mathrm{n} 2} \sqcup \mathrm{OSf}=\mathrm{n}_{\mathrm{n} 3}\right] .
$$

A formalized description of the SODA stages is presented in the table.

\section{Description of the Stages of System-Object Determinant Analysis}

The first stage of SODA has been developed in detail - the construction of a generic classification (taxonomy) or conceptual system that defines the abstract requirements for the system being designed at the class level. The results are presented in [17].

The procedure for constructing a taxonomy of a subject area is concluded to three steps.

1. Selection and formulation of a generic definition of the most general concept/class of the subject area $\left(S_{1,1}{ }^{1}\right)$. This definition should reflect in the specific difference $\left(R S_{1,1}{ }^{1}\right)$ the functional properties of an object or phenomenon corresponding to the defined concept/class $\left(S_{1,1}{ }^{1}\right)$. Ideally, these should be properties holding the functional properties $\left(R S_{0,1}{ }^{0}\right)$ of the concept/class $\left(S_{0,1}{ }^{0}\right)$ which is generic for the chosen general concept/class $\left(S_{1,1}{ }^{1}\right)$ subject area. Thus, the specific difference of the general concept/class of the subject area $\left(R S_{1,1}{ }^{1}\right)$ must be a kind of species difference $\left(R S_{0,1}{ }^{0}\right)$ of a 
concept/class $\left(S_{0,1}{ }^{0}\right)$ generic for the chosen concept/class $\left(S_{1,1}{ }^{1}\right)$. Based on expression (1) we can write that $S_{1,1}{ }^{1}=S_{0,1}{ }^{0} \sqcap \exists R S_{1,1}{ }^{1}$, where $S_{1,1}{ }^{1} \sqsubset S_{0,1}{ }^{0}$ и $R S_{1,1}{ }^{1} \sqsubset R S_{0,1}{ }^{0}$.

2. Decomposition of the general concept/class of the subject area into specific concepts/classes for which the general concept/class is generic. So $S_{2,1}{ }^{1}, \ldots S_{2, n}{ }^{1} \sqsubset S_{1,1}{ }^{1}$. In this case, the properties of specific concepts/classes reflected in their specific differences must be supportive for the functional properties of the general concept/class. Thus, the species differences of the species concepts/classes should be the species differences of the general concept/class. That is $R S_{2,1}{ }^{1}, \ldots R S_{2, n}{ }^{1} \subset R S_{1,1}{ }^{1}$. Based on the expression (2) we can write that $S_{2, j}{ }^{1}=S_{1,1}{ }^{1} \sqcap \exists R S_{2, j}{ }^{1}$, where $S_{2, j}{ }^{1} \sqsubset S_{1,1}{ }^{1}$ и $R S_{2, j}{ }^{1} \sqsubset$ $R S_{1,1}{ }^{1}$

3. Repetition of step 2 for each species concept/class $\left(S_{2,1}{ }^{1}, \ldots, S_{2, n}{ }^{1}\right)$. Those definition of systems-classes of the $\mathrm{i}$-th level through the classes of the upper (in this case, the 2nd) level, for example, $S_{i, j}{ }^{l}=S_{2,1}{ }^{1} \sqcap \exists R S_{i, j}{ }^{l} ; \ldots ; S_{i, p}{ }^{l}=S_{2, n}{ }^{1} \sqcap \exists R S_{i, p}{ }^{l}$, such that for their properties-classes $R S_{i, j}{ }^{l}, \ldots, R S_{i, p}{ }^{l} \subset R S_{2,1}{ }^{1}$ (fig. 1).

The procedure for constructing a taxonomy of classes can be described as abstract systems-classes ends with the appearance at a certain level of the hierarchy of a class which is a specific system-class that can be described using classes of links (flows), classes of functions (processes) and classes of objects (object characteristics).

The concept/class is the result of the classification of the subject area. It includes the analyzed system in which the functional properties of this system are generally determined. The definition of the class of links, in fact, is the definition of a functional request (external determinant) of the analyzed or designed system and the definition of object characteristics specifies the ways of implementing the functions of the system.

The construction of a genetic classification is presented as a procedure consisting of the following steps:

1. After the stage of constructing a generic classification we have a specific systemclass which includes the projected system, of the following form: $S_{i+1, j} I^{j 0}=\left[L ?_{i+1, j I^{j}}\right.$

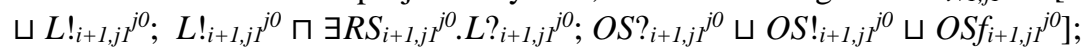

2. We clarify the node of the system by introducing specific sets of units instead of classes of units using the concept of nominal: $S_{i+2, j 2^{j l}}=\left[\left\{\mathrm{L} ?_{i+2, j 2^{j}}\right\} \sqcup\left\{\mathrm{L} !_{i+2, j 2^{j l}}\right\}\right.$; $\left.\left\{\mathrm{L} !{ }_{i+2, j 2^{j}}\right\} \sqcap \exists R S_{i+2, j 2^{j l}} .\left\{L ?_{i+2, j 2^{j}}{ }^{l}\right\} ; O S ?_{i+2, j 2^{j l}} \sqcup O S !_{i+2, j 2^{j l}} \sqcup O S f_{i+2, j 2^{j}}\right]$;

3. We clarify the function of the system by defining the internal determinant of the designed system as a set of functions instead of a class of functions: $S_{i+3, j}{ }^{2}=$

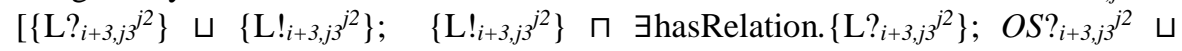
$\left.O S !_{i+3, j 3^{j}} \sqcup O S f_{i+3, j 3^{2}}{ }^{2}\right]$.

4. We clarify the object of the designed system using the concept of specific areas: $s_{i+4, j 4^{j 3}}=\left[\left\{\mathrm{L} ?_{i+4, j 4^{j 3}}\right\} \cup\left\{\mathrm{L} !_{\left.i+4, j 4^{j 3}\right\} ;}\right\} ; \mathrm{L} !_{i+4, j 4^{j 3}}\right\} \quad \Pi \quad \exists$ BhasRelation. $\left\{L ?_{i+4, j 4^{j 3}}\right\} ;$ $\left.\operatorname{OS} ?_{i+4, j 4^{j 3}} .{ }_{n 1} \sqcup \mathrm{OS} !_{i+4, j 4^{j 3}} .{ }_{n 2} \sqcup \operatorname{OSf}_{i+4, j 4^{j 3}} .{ }_{n 3}\right] ; n 1, n 2, n 3$ - the values of the corresponding fields of the object (attributes). 
Table 1. Formalized SODA stages

\begin{tabular}{|c|c|}
\hline $\begin{array}{l}\text { Meaningful in- } \\
\text { terpretation }\end{array}$ & SODA stages \\
\hline $\begin{array}{l}\text { Defining abstract } \\
\text { requirements at the } \\
\text { class level }\end{array}$ & 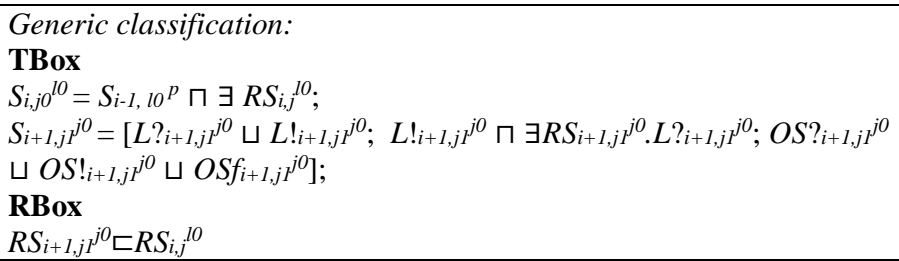 \\
\hline $\begin{array}{c}\text { Specifying require- } \\
\text { ments at the set } \\
\text { level }\end{array}$ & 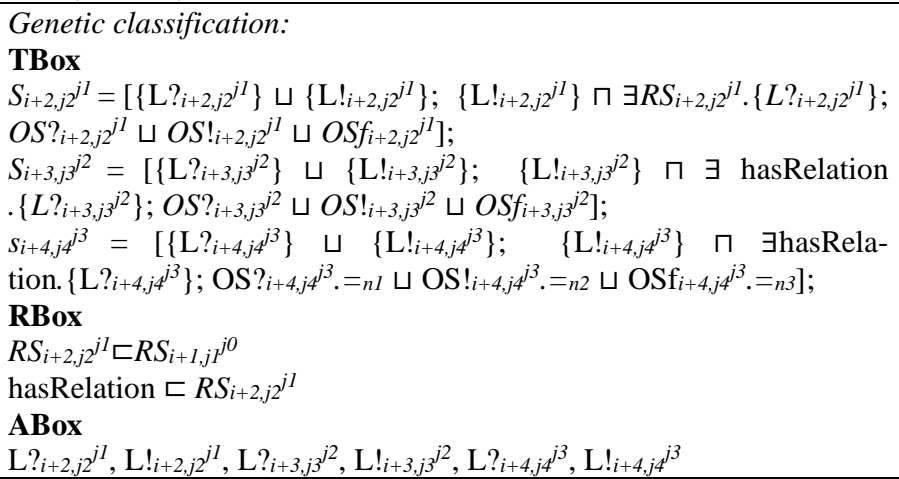 \\
\hline $\begin{array}{l}\text { Description of the } \\
\text { project that ensures } \\
\text { the implementation } \\
\text { of the requirements }\end{array}$ & 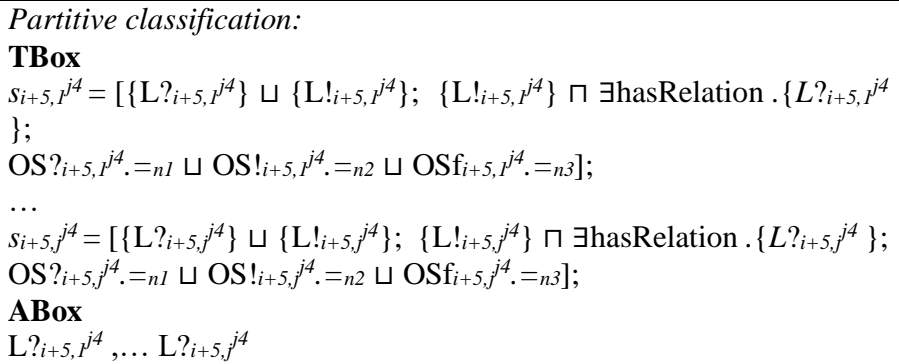 \\
\hline
\end{tabular}

To construct a partitive classification (meronomy) a formal-semantic normative system has been developed presented in [17]. The normative system $N$ is based on a formal-semantic alphabet which consists of unambiguously interpreted symbols from the point of view of generic relations. The alphabet is based on the classification of the concepts of connections/flows which in the notation accepted in the DL can be represented as follows: $\mathbf{M} \sqsubset \mathbf{L}, \mathbf{I} \sqsubset \mathbf{L}, \mathbf{V} \sqsubset \mathbf{M}, \mathbf{E} \sqsubset \mathbf{M}, \mathbf{D} \sqsubset \mathbf{I}, \mathbf{C} \sqsubset \mathbf{I}$. Here $\mathbf{L}$ is the whole set of connections/flows, $\mathbf{M}$ is the set of material connections/flows, $\mathbf{I}$ is the set of information connections/flows, $\mathbf{V}$ is the set of material connections/flows, $\mathbf{E}$ is the set of energy connections/flows, $\mathbf{D}$ is the set of connections/flows of data, $\mathbf{C}$ is the set of connections/flows of control. In this case, further division of the presented links into subspecies is assumed if necessary.

The alphabet of links is used to describe the alphabet of nodes as crossroads of alphabetic links. The alphabet of nodes is specified using the following set of rules for each type of link:

$\mathbf{V} \equiv \mathbf{v} ! \sqcap$ ヨhasRelation.(v?); 


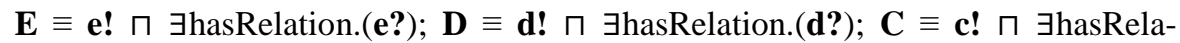

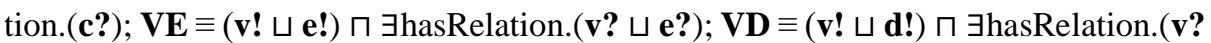

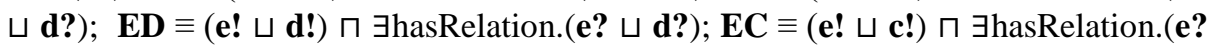
$\sqcup \mathbf{c}$ ?); DC $\equiv(\mathbf{d}$ ! $\sqcup \mathbf{c} !) \sqcap$ ヨhasRelation.(d? $\sqcup \mathbf{c} ?)$.

The presented rules for constructing the alphabet of the normative system using the classification scheme ensure its subject (problem) orientation which makes this normative system formal-semantic and expandable depending on the analyzed subject area.

The construction of a partitive classification (meronomy) is described with the following steps:

1. Let $s_{i+4, j 4^{j 3}}=\left[\left\{\mathrm{L} ?_{i+4, j 4^{j 3}}\right\} \sqcup\left\{\mathrm{L} !_{i+4, j 4^{j 3}}\right\} ; \quad\left\{\mathrm{L} !_{i+4, j 4^{j 3}}\right\} \sqcap\right.$ ヨhasRelation. $\left\{L ?_{i+4, j 4^{j 3}}\right\}$; $\left.\mathrm{OS} ?_{i+4, j 4^{j 3} .}={ }_{n 1} \sqcup \mathrm{OS} !_{i+4, j 4^{j 3}} .={ }_{n 2} \sqcup \mathrm{OSf}_{i+4, j 4^{j 3}} .{ }_{n 3}\right]$ system-phenomenon; We perform system decomposition $s_{i+4, j 4^{j 3}}$ into parts $(j 4=1, N)$ whose functional properties are hold up the system $s_{i+4, j 4^{j 3}}$.

2. For each subsystem $s_{i+5, j}{ }^{j 4}=\left[\left\{\mathrm{L} ?_{i+5, j}{ }^{j 4}\right\} \sqcup\left\{\mathrm{L} !_{i+5, j}{ }^{j 4}\right\} ; \quad\left\{\mathrm{L} !_{i+5, j}{ }^{j 4}\right\} \sqcap \exists\right.$ hasRelation. $\left\{L ?_{i+5, j}{ }^{j 4}\right\}$; OS $\left.?_{i+5, j}{ }^{j 4} .={ }_{n 1} \sqcup \mathrm{OS} !_{i+5, j}{ }^{j 4} .{ }_{n 2} \sqcup \mathrm{OSf}_{i+5, j}{ }^{j 4} .{ }_{n 3}\right]$ we determine the types of links and nodes based on the previously introduced normative system.

3. Repetition of step 2 for all subsystems of systems-phenomena $s_{i+5, j}{ }^{j 4}$.

Thus, the final result of all stages of SODA in terms of DL can be represented as $\mathbf{K}$ $=$ TBox $\cup$ RBox $\cup$ ABox. In general form, the constituent elements of $\mathrm{K}$ are represented as follows:

$$
\begin{aligned}
& \operatorname{RBox}=\left[\begin{array}{l}
R S_{i+1, j 1}{ }^{j 0} \sqsubset R S_{i, j}{ }^{l 0} \\
R S_{i+2, j 2}{ }^{j 1} \sqsubset R S_{i+1, j 1}{ }^{j 0} \\
\text { hasRelation } \sqsubset R S_{i+2, j 2}{ }^{j 1} \\
\ldots
\end{array}\right] ; \text { ABox }=\left[\begin{array}{l}
L ?_{i, j}{ }^{l} \\
L !_{i, j}{ }^{l} \\
\ldots
\end{array}\right] \text {; }
\end{aligned}
$$

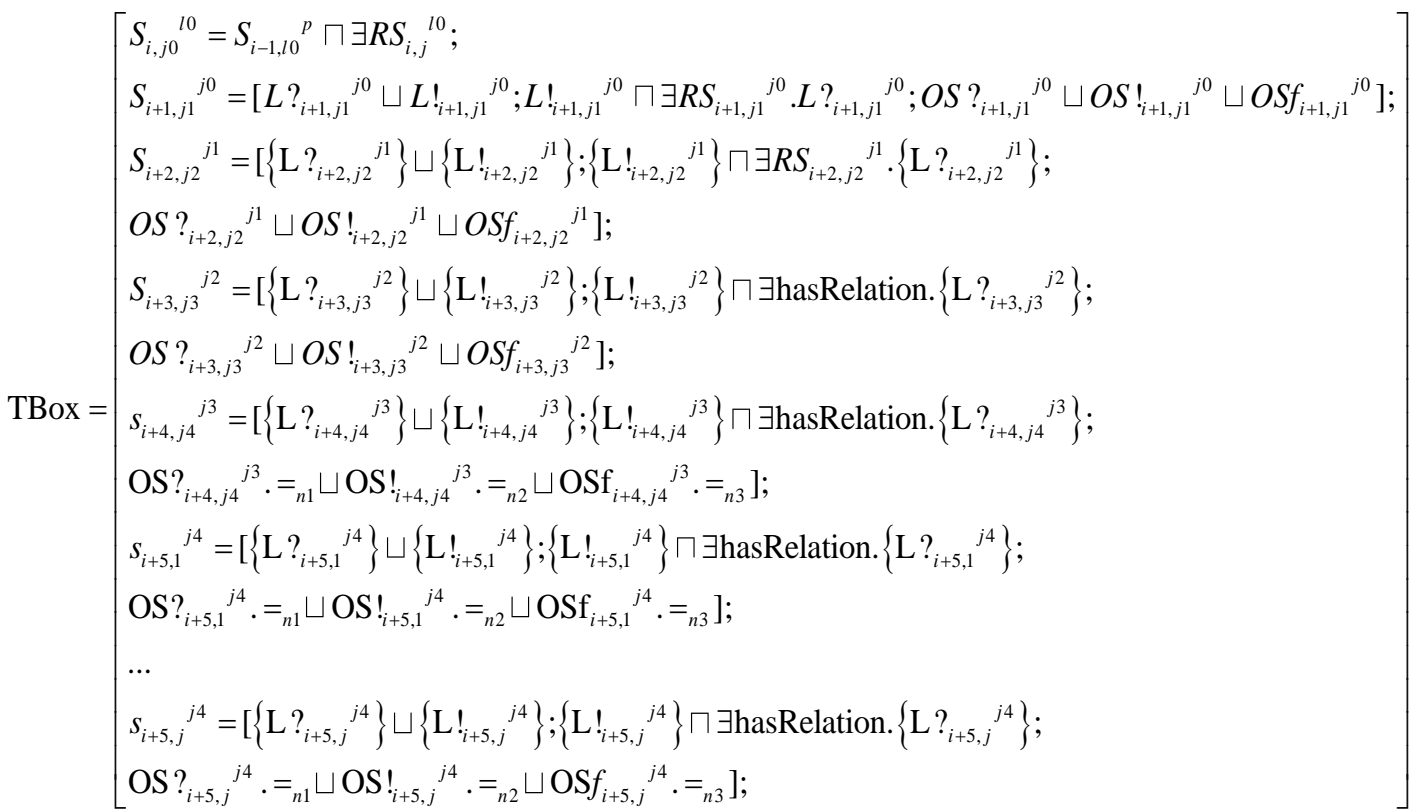




\section{An Example of the Applying of the Stages of SODA}

Below is an example of a subject area taxonomy for car classification.

\section{Generic classification stage.}

- Car $\left(S_{1,1}^{l}\right)$ - a vehicle $\left(S_{0,1}^{l}\right)$, for the carriage of passengers and luggage with a capacity of 2 to 8 people $\left(R S_{l, l}{ }^{l}\right)$.

- Passenger $\left(S_{2,1}^{l}\right)$ - a car $\left(S_{1,1}^{l}\right)$ for paved roads $\left(R S_{2,1}^{l}\right)$.

- Sport $\left(S_{3,1}^{l}\right)$ - a passenger car $\left(S_{2,1}^{l}\right)$ for dynamic driving $\left(R S_{3,1}^{l}\right)$.

- Executive $\left(S_{3,2^{l}}\right)$ - a passenger car $\left(S_{2,1}{ }^{l}\right)$ to ensure increased comfort of passengers $\left(R S_{3,2}^{l}\right)$.

○ Crossovers $\left(S_{3,3^{l}}\right)$ - a passenger car $\left(S_{2, I^{l}}\right)$ for paved and unpaved roads $\left(R S_{3,3}{ }^{l}\right)$.

- Especially small $\left(S_{4,1^{3}}\right)$ - a crossover with a length of less than $4241 \mathrm{~mm}$., a width of less than $1765 \mathrm{~mm}$., a wheelbase of less than $2591 \mathrm{~mm}$., an engine capacity of less than 1.6 liters., an engine power of less than $120 \mathrm{hp}$;

Let's represent the resulting concrete system-class $\mathrm{S} 4,13$ as (2)

$S_{4,1}{ }^{3}=[L ? \sqcup L ! ; L ! \sqcap \exists R S . L ? ; O S ? \sqcup O S ! \sqcup O S f]$,

$L$ ? = passengers $\sqcup$ luggage $\sqcup$ consumables $\sqcup$ control actions

$L !$ = passengers $\sqcup$ luggage $\sqcup$ torque $\sqcup$ replaceable parts $\sqcup$ effects from control actions

$R S=$ torque provision $\sqcup$ movement of passengers and luggage on roads with or without paved roads $\sqcup$ maintenance

$O S ? \sqcup O S !=$ overall characteristics of input and output elements

OSf = volumetric characteristics for storage of materials and objects) $\sqcup$

$\sqcup$ length. $\leq 4241 \mathrm{Mm}$. $\sqcup$ width. $\leq 1765 \mathrm{Mm}$. $\sqcup$ wheelbase. $\leq 4241 \mathrm{Mm}$. $\sqcup$ (engine capacity). $\leq 1.6$ л. $\sqcup$ (engine power).$\leq 120$ л.с.

Genetic classification stage. We concretize the system $S_{4,1} 3$ in the shape of (3):

$s_{5,1}{ }^{1}=\left[\{\mathrm{L} ?\} \sqcup\{\mathrm{L} !\} ;\{\mathrm{L} !\} \sqcap \exists \mathrm{RS} .\{\mathrm{L} ?\} ; \mathrm{OS} ? .={ }_{\mathrm{n} 1} \sqcup \mathrm{OS} ! .={ }_{\mathrm{n} 2} \sqcup \mathrm{OSf} .=_{\mathrm{n} 3}\right]$

Refining the unit

$\{$ L $\}=5$ passengers $\sqcup$ luggage $\sqcup 95$ gasoline $\sqcup$ driver control

$\{\mathrm{L} !\}=5$ passengers $\sqcup$ luggage $\sqcup$ torque $\sqcup$ effects from control actions

Refining the function

$R S=$ torque provision $\sqcup$ movement of passengers and luggage on roads with or without paved roads $\sqcup$ maintenance

Refining the object

$O S ?=($ passenger weight $) .=80$ кг. $\sqcup($ baggage weight $) .=250$ кг.

$O S !=($ passenger weight $) .=80$ кг. $\sqcup$ (baggage weight $).=250$ кг.

$O S f=$ volumetric characteristics for storage of materials and objects $\sqcup$

$\sqcup$ length. $=_{4220 \text { мм. }} \sqcup$ width. $={ }_{1750 \text { мм. }} \sqcup($ wheelbase $) .={ }_{4220 \text { мм. }} \sqcup($ engine capacity $) .={ }_{1.6 л . ~} \sqcup$ (engine power).$=120$ л.с. 
Partitive classification stage. System $s_{5,1}^{l}$ decompose into subsystems: Engine $\left(s_{6,1}{ }^{l}\right)$ ᄃ $s_{5,1}^{l}$; Chassis $\left(s_{6,2}{ }^{l}\right) \sqsubset s_{5,1}^{1}$; Control system $\left(s_{6,3}^{l}\right) \sqsubset s_{5,1}^{l}$;

We use the previously given normative system $N$ and build a decomposition diagram of the process of driving a car: Engine (CE), Control system (CD), Chassis (VE). See Fig.1. This diagram is built in the "Unit-Function-Object" notation in the UFO-toolkit software environment which implements the system-object approach.

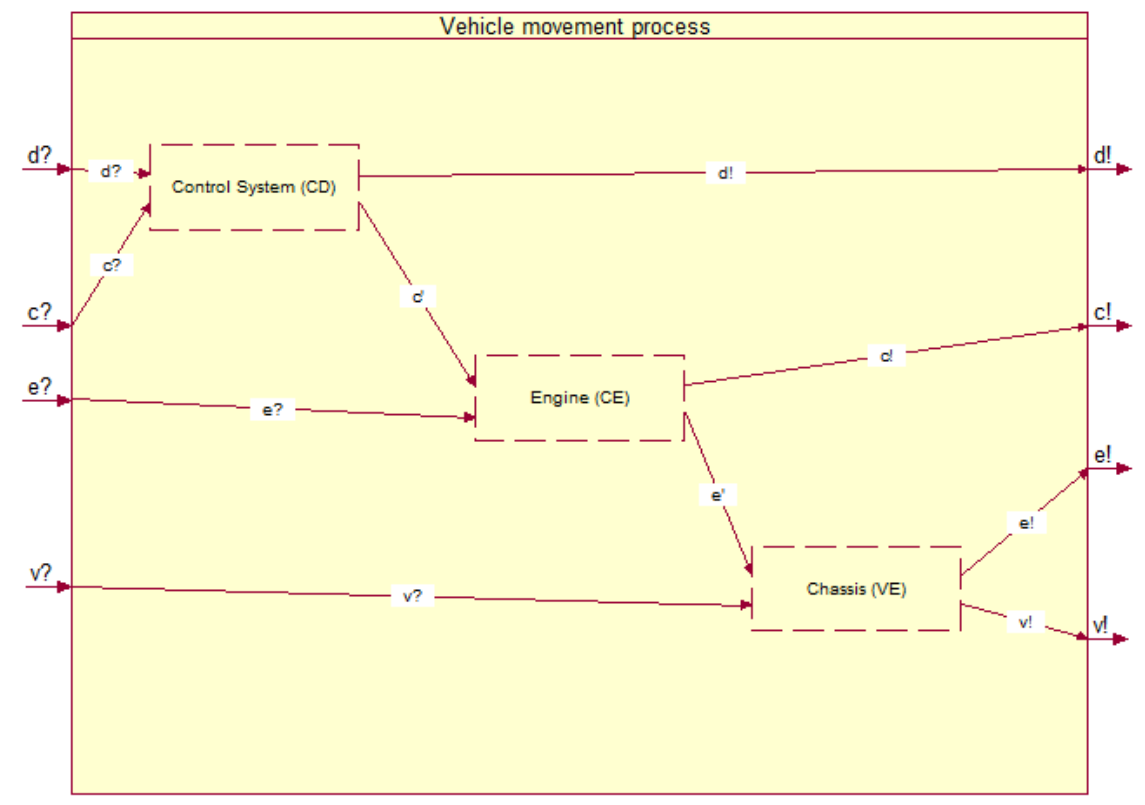

Fig. 1. Diagram of the decomposition of the process of vehicle movement, drawed in the UFOtoolkit software environment

Then you can carry out further decomposition of each of the subsystems $\left(s_{6,1}{ }^{1}, s_{6,2}{ }^{1}\right.$, $\left.s_{6,3^{l}}\right)$.

\section{Conclusion}

The system-object determinantant analysis presented in the paper provides the researcher a new toolkit for the analysis and design of complex weakly formalized systems which is a formalized procedure that takes into account system-wide patterns (in contrast to the existing methods of system analysis).

Identifying the class to which the analyzed system belongs gives the researcher an idea of the purpose of the system. Also makes it possible to clarify the requirements for the system by describing specific classes of inputs and outputs of the system and a specific class of transformation of inputs into outputs and specific classes of object characteristics. This allows you to unambiguously determine the external determinant 
of the system. The formulation of a set of requirements for a specific class of such systems actually formalizes the process of creating a technical assignment for the development of a new technical or information system.

Moreover, tracing the stages of formation (or creation) of a system allows to concretize the requirements for the system to the level of describing specific input and output streams as phenomena, specific functional requirements and specific object characteristics. On the other hand, allows to unambiguously determine the internal determinant of the system.

The decomposition of the requirements for the system as a phenomenon (or its internal determinant) gives the analyst or the developer an idea of how to ensure the compliance of subsystems with the analyzed or developed system with its internal determinant, i.e. ways of functioning or building a system.

The proposed system analysis technique (SODA) is based on the use of knowledge models: a conceptual model and a process model. The future toolkit supporting SODA will therefore be a knowledge-based system, i.e. intelligent information system.

Thus, the described method of analyzing and representing systems using the means of system-object determinant analysis will be useful and promising in the design of poorly formalized information and technical systems.

\section{References}

1. Kachala V.V. General systems theory and systems analysis. Hot line. Telecom, Moscow (2017).

2. Surmin Yu.P. Systems theory and systems analysis. MAUP, Kiev (2003).

3. Spitsnadel V.N. Fundamentals of systems analysis. "Publishing house" Business-press, Saint-Petersburg (2000).

4. Volkova V.N., Emelyanov A.A. Systems Theory and Systems Analysis in Organizational Management: A Handbook. Finance and statistics, Moscow (2006).

5. Volkova V.N., Denisov A.A. Systems theory and systems analysis. Yurayt, Moscow (2015).

6. Matorin S.I., Zhikharev A.G., Zimovets O.A., Systems theory and systems analysis: textbook. Directmedia Publishing, Moscow (2020).

7. Quaid E.S. Analysis of complex systems. Translated from English by I.M. Vereshchagin, A.G. Lebedeva, N.A. Malyarshchikova, G.M. Smakhtin edited by I.I. Anureeva, I.M. Vereshchagin. Soviet Radio, Moscow (1969)

8. Optner S. System analysis for solving business and industrial problems. Soviet radio, Moscoew (1969)

9. Chernyak Yu.I. System analysis in economic management. Economics, Moscow (1975)

10. Golubkov E.P. The use of systems analysis in sectoral planning. Economics, Moscow (1977)

11. Young S. Systemic management of the organization. Translated from English ed. S. P. Nikanorova, S. A. Batasova. M., Soviet Radio, Moscow (1972)

12. Peregudov F.I., Tarasenko F.P. Introduction to systems analysis. Higher school, Moscow (1989)

13. Matorin S.I., Mikhelev V.V. System-Object Approach to the Determinant Analysis of Complex Systems. In: Artificial Intelligence and Decision Making No. 2, pp. 86-93. Moscow (2020).

14. Melnikov G.P. Systemology and linguistic aspects of cybernetics. Sov. radio, Moscow (1978). 
15. Baader F., Calvanese D., McGuinness L., Nardi D. Patel-Schneider P. F. The Description Logic Handbook: Theory, Implementation, and Applications. Cambridge University Press, Cambridge (2003).

16. Matorin S.I., Zhikharev A.G., Zimovets O.A. System theory and system analysis. KNORUS, Moscow (2021).

17. Matorin S.I., Mikhelev V.V. System-object determinant analysis. 1. Building a subject area taxonomy. Artificial intelligence and decision making No. 1. Moscow (2021).

18. Matorin S.I., Mikhelev V.V., Zhikharev A.G. Normative system of system-object analysis and modeling, pp. 623-637. Economics. Informatics No. 3., Moscow (2020). 